\title{
Mature Cystic Retroperitoneal Teratoma with Well Differentiated Renal Elements: Relation to Spinal Dysraphism
}

\author{
Anand Sinha ${ }^{1}$ Arvind Sabharwal ${ }^{1}$ Nandini Yadav ${ }^{2}$ \\ ${ }^{1}$ Department of Pediatric Surgery, Fortis Memorial Research Institute, \\ Gurgaon, Haryana, India \\ 2 Department of Pathology, Fortis Memorial Research Institute, \\ Gurgaon, Haryana, India \\ ${ }^{3}$ Department of Radiodiagnosis, Fortis Memorial Research Institute, \\ Gurgaon, Haryana, India \\ ${ }^{4}$ Department of Neurosurgery, Fortis Memorial Research Institute, \\ Gurgaon, Haryana, India
}

\author{
R. K. Gupta ${ }^{3}$ Rana Patir ${ }^{4}$ \\ Address for correspondence Anand Sinha, MCh, Pediatric Surgery, \\ Department of Pediatric Surgery, Fortis Memorial Research Institute, \\ Sector 44, Gurgaon, Haryana 122002, India \\ (e-mail: dranandsinha@hotmail.com).
}

Eur J Pediatr Surg Rep 2014;2:46-49.

\begin{abstract}
Keywords

- retroperitoneal teratoma

- mature renal elements

- intraspinal extension

Retroperitoneum is a relatively uncommon site for pediatric teratomas. Rarely, such tumors can have an intraspinal extension and few cases of retroperitoneal teratomas associated with spinal dysraphism have been reported. Teratomas consist of tissues arising from all three embryonic layers. However, mature renal tissues in the form of glomeruli and tubules are sparingly found in teratomas. A 15-day-old female presented with spina bifida occulta and on evaluation a cystic presacral mass was detected. Intraoperatively the cyst was found densely adherent to the hemivertebrae but not entering the spinal canal. Histopathological examination confirmed a mature cystic teratoma but also demonstrated presence of mature renal elements in the cyst wall. The teratomas lying in proximity to spine and associated with spinal dysraphism are likely to contain mature renal tissues or even nephroblastic elements. It supports the dysembryogenic model of origin of intradural teratomas from native progenitor cells rather than aberrantly migrated germ cells.
\end{abstract}

\section{Introduction}

Retroperitoneum is a relatively uncommon site for pediatric teratomas accounting for 3 to $5 \%$ for all teratomas in prepubertal children. ${ }^{1}$ Most of these teratomas lie separate from the spine. However, very rarely, such tumors can have an intraspinal extension. ${ }^{2}$ Intraspinal teratomas are also rare. ${ }^{3} \mathrm{Few}$ cases of retroperitoneal teratomas associated with spinal dysraphism have been reported. ${ }^{4}$

Teratomas consist of tissues arising from all three embryonic layers. However, mature renal tissues in the form of glomeruli and tubules are sparingly found in teratomas. This study reports a case of a mature cystic retroperitoneal teratoma associated with spinal dysraphism harboring mature renal elements. In the review of literature, the authors found that the teratomas lying in proximity to spine and associated with spinal dysraphism are likely to contain mature renal tissues or even nephroblastic elements.

\section{Case Report}

A 15-day-old female presented with a swelling in the lumbosacral region since birth. Antenatal ultrasounds had revealed mild right sided hydroureteronephrosis. On examination, the baby had $4 \times 3 \mathrm{~cm}$ soft, vague protuberance in the lumbosacral region of the spine with a hemagiomatous patch on the overlying received

April 3, 2013

accepted after revision

June 23, 2013

published online

August 5, 2013

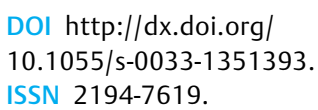

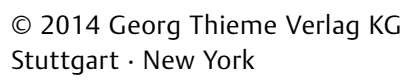

License terms

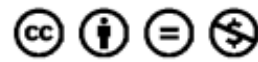




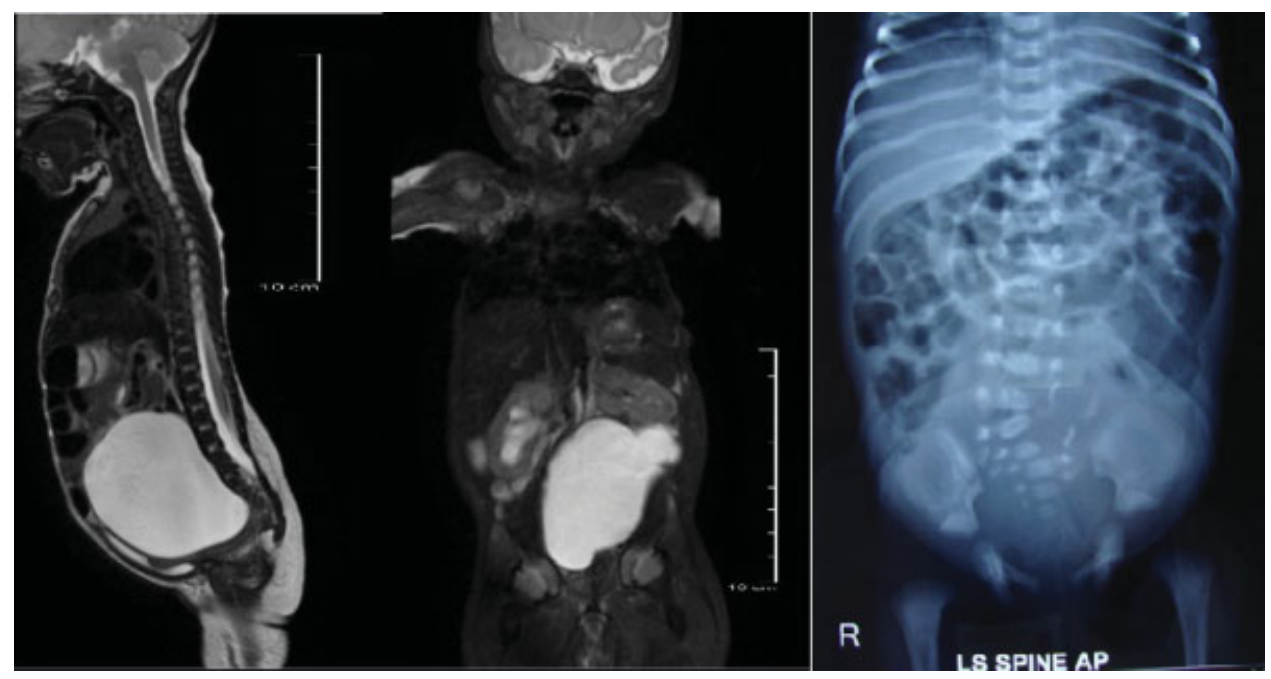

Fig. 1 Magnetic resonance imaging demonstrated a large cystic presacral mass without any intraspinal extension.

skin, a sacral pit, and tuft of hair below the swelling. In addition the child had a tense cystic lump occupying almost the entire abdomen, predominantly the left lumbar, left hypochondrium, left iliac fossa, umbilical, and hypogastric region and extending onto the right side. On per rectal examination the sacrum was free and the mass was palpated anterolateral to the rectum. Bilateral lower limb movements and anal tone were normal. The $\mathrm{X}$-ray of the spine revealed hemivertebrae of $\mathrm{L} 5 \mathrm{~S} 1$ vertebrae. The ultrasound detected a cystic presacral mass. The magnetic resonance imaging (MRI) for the abdomen, pelvis, and spine was done and revealed an $8 \times 6 \times 4 \mathrm{~cm}$ cystic mass arising from the presacral region, reaching very close to the sacral promontory but the dural sac was found to be intact. The mass had pushed the bowel loops and the sigmoid colon to the right and it was pushing on the inferior pole of the left kidney, thereby altering its position into a transverse one ( - Fig. 1). The serum $\alpha-$ feto protein was $3,574 \mathrm{ng} / \mathrm{dL}$, which was normal for her age. With the differential diagnosis of an ovarian cyst or benign cystic retroperitoneal teratoma, the child was posted for laparoscopic excision of the cyst.
On laparoscopy, the cyst was found arising from behind the uterus. Approximately $90 \mathrm{~mL}$ of clear fluid was aspirated to facilitate dissection. The cyst could be mobilized in all directions except inferiorly where it was found closely adherent to the spine. In view of the possibility of the cyst entering the spinal canal (especially in the presence of the vertebral anomalies), a small Pfannenstiel incision was made and further dissection was done under an operating microscope. The cyst wall was adherent but not entering the vertebral bodies and was shaved off from the sacral promontory. The cyst was excised in toto. The right ovary was well developed but the left ovary could not be defined.

Histopathological examination revealed a large cystic swelling with a small solid nodule adherent to the wall. The cyst wall showed features of mature teratoma but the nodule consisted of well-formed and abortive glomeruli and tubules. No ovarian stroma was seen ( - Fig. 2). The final diagnosis was a mature cystic retroperitoneal teratoma with mature renal elements along with spinal bifida occulta (L5, S1 hemivertebrae).

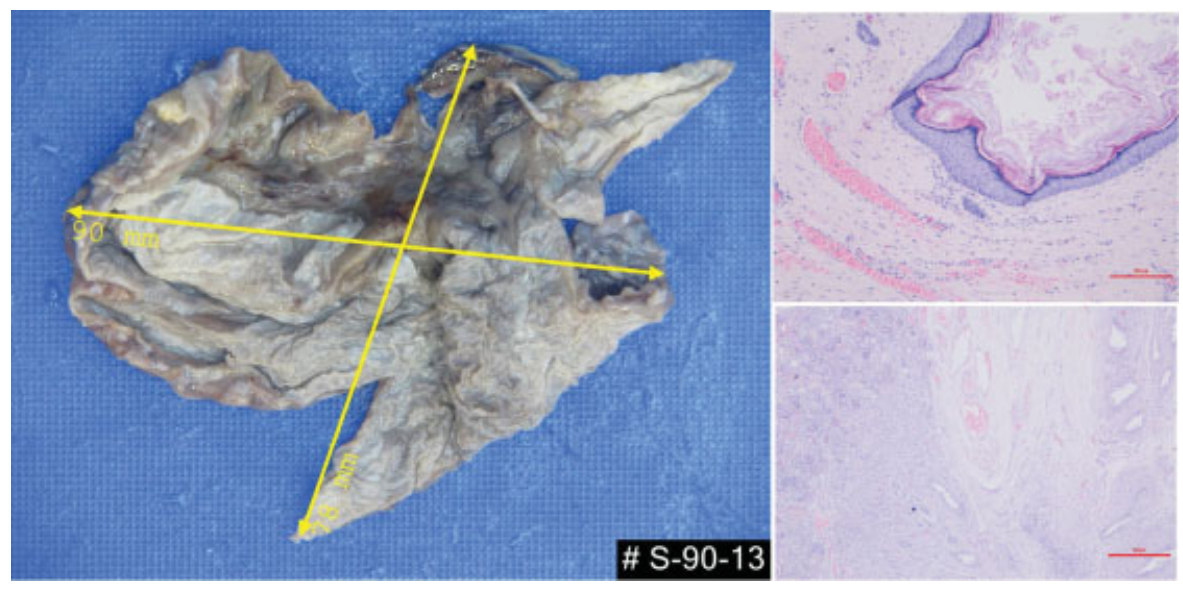

Fig. 2 Histopathology demonstrated a large cystic teratoma with mature renal elements in the wall (inset). 


\section{Discussion}

This case posed a diagnostic dilemma at various stages. Retroperitoneum is a rare site for extragonadal germ cell tumors (GCTs) in children accounting for 3 to $5 \%$ cases of all germ cell tumors. ${ }^{1}$ Grossly, teratomas can be of two types: (1) cystic teratomas, usually benign, containing yellowish liquid and material resembling hair and composed of fully developed tissues (2) solid teratomas, formed of fibrous, fatty, cartilaginous, and bone tissue. ${ }^{5}$ A completely cystic teratoma arising in the retroperitoneum is comparatively rare. ${ }^{6}$ Hence, an ovarian cyst was considered to be the first possibility and a laparoscopic excision of the cyst was planned. There have been case reports of cystic teratomas resembling ovarian cysts. Cho et $\mathrm{al}^{7}$ reported an incidentally found pelvic mass, considered to be an ovarian tumor by ultrasonography in a 27-year-old female. In the adults, gonadal teratomas are far more common than extragonadal ones. MRI strongly suggested a retroperitoneal cystic tumor because of anterior displacement of retroperitoneal organs. The large retroperitoneal mature cystic teratoma was successfully resected laparoscopically. A large mature cystic retroperitoneal teratoma can mimic a far more common ovarian cyst in any age group.

Since a vertebral anomaly was the predominant presenting complaint, the initial differential also included all the masses which are known to have a dumbbell-shaped extension into the spine. Neuroblastoma, anterior meningoceles, and neuroenteric cysts can all present as presacral lumps with intraspinous extension. Neuroblastomas are usually solid but cystic variants have been described. ${ }^{8}$ However, the ones entering the spinal canal are usually solid. Anterior sacral meningoceles are a very rare form of spinal dysraphism characterized by protrusion of dural sac through an anterior defect in the sacrum. There have been very few case reports in children describing this anomaly. ${ }^{9}$ Even rarer is neuroenteric cysts in the lumbosacral region with fewer than 10 instances in literature. ${ }^{10-12}$ However, these can present as cystic masses with dumbbell-shaped extension into the spine and are mostly associated with vertebral defects. Some other soft tissue sarcomas have also been known to have an intraspinous extension. However, they are usually solid. ${ }^{13}$ The occurrence of intradural spinal teratomas in association with spinal dysraphism is also rare. ${ }^{14}$ Teratomas have known to be purely retroperitoneal, entirely intraspinal ${ }^{3,14-16}$ or can also present as a presacral masses with intradural extension. ${ }^{2}$

Retroperitoneal or extragonadal location of teratomas is commonly explained by aberrant migration of germ cells. At the fourth week of gestation, pluripotent germ cells migrate from the yolk sac to the fetal midline to reach the gonads. A few of them can get arrested and survive at extragonadal sites, mainly the dorsal midline. This theory explained the location of extragonadal germ cell tumors in anterior mediastinum, retroperitoneum, intracranial, and sacrococcygeal location. ${ }^{17}$ However, various studies support a dysembryogenic model of development of some these extragonadal teratomas especially intradural teratomas. The caudal spinal cord in the developing embryo contains a population of undifferentiated mesenchymal stem cells which can give rise to teratomas when transplanted into developing embryos. ${ }^{18}$ The developing neural tube and adjacent somites establish signaling centers that guide the differentiation of adjacent tissues and disruption of signaling centers can affect the migration and patterning of progenitor cells, resulting in their retention and the formation of teratomas. ${ }^{18}$ This is further supported by the fact that many of these tumors are associated with spinal dysraphism. $^{14,16}$

In this case, the MRI clearly excluded any intraspinous communication but the baby had hemivertebrae L5 S1. Intraoperatively, the teratoma was only found to be closely adhered to sacral promontory and not entering the spinal canal. Histopathology concluded the mass to be a cystic mature teratoma but with well-differentiated renal tissue along with the cyst. Renal differentiation in a teratoma is even rarer.

There have been very few reports of teratomas with welldifferentiated mature renal tissue. Su et $\mathrm{al}^{19}$ also reported a mixed retroperitoneal GCT in an 11-year-old girl presenting with renal hypertension. Jain et al ${ }^{14}$ reported two patients (1$\mathrm{y}$-old boy and 3-y-old girl) with spinal dysraphism, split cord malformation (SCM), and intraspinal teratomas with renal differentiation. Surgical excision of the mass, detethering of the cord, and repair of the lipomyelomeningocele was done and pathological examination in both the cases revealed features of a mature teratoma with nephrogenic differentiation in the form of elements of mature renal tissue. Uzum et $\mathrm{al}^{20}$ have also reported a case of intradural spinal teratoma with SCM, tethered cord, and immature nephroblastic tissue. In this case, the cyst was not intraspinal but was closely adhered to the vertebrae and was associated with spinal dysraphism. This supports the dysembryogenesis model of formation of teratomas lying within or in close proximity to spinal cord. The authors also support the theory that neural tube defects interfere with the migration of renal tissue during embryogenesis resulting into renal differentiation within the teratoma.

Extrarenal Wilms tumor can also rarely arise within a teratoma. Interestingly most of these reports in literature describe teratomas which were in close proximity to the spinal cord. Kim et al in 1990, reported a 10-month-old girl with a retroperitoneal teratoma with nephroblastic components. ${ }^{4}$ The girl also had spinal dysraphism. They also reviewed the literature and found five similar cases with teratomas harboring nephroblastic elements. Surprisingly, all five of these cases were extragonadal and in close proximity to the spinal column (two retroperitoneum, two sacrococcygeal, and one posterior mediastinum).

\section{Conclusions}

Teratomas found in close proximity to or within spinal canal are likely to be associated with spinal dysraphism due to a likely common dysembryogenic model of origin. During development, these neural tube defects are likely to interfere with renal differentiation and hence commonly harbor mature renal tissues or nephroblastic elements. Such teratomas 
are more likely to arise from native pluripotent cells rather than aberrantly migrated germ cells.

\section{Conflict of Interest}

None.

\section{References}

1 Shamberger RC. Teratomas and germ cell tumors. In: O'Neill JA Jr, Grosfeld JL, Fonkalsrud EW, et al eds. Principles of Pediatric Surgery, 2nd ed. St Louis: Mosby; 2004

2 Kao TH, Shen CC, Chen CC, Kwan PH. "Primary" benign retroperitoneal and intraspinal dumbbell-shaped cystic teratoma: case report. Spine 2005;30(15):E439-E443

3 Kalani MY, Iyer S, Coons SW, Smith KA. Spinal intradural teratomas: developmental programs gone awry? Neurosurg Focus 2012; 33(4):E1

4 Kim YW, Park YK, Oh SM, Yang MH. Retroperitoneal teratoma with predominance of nephroblastic elements-a case report. J Korean Med Sci 1990;5(4):237-242

5 Bruneton JN, Diard F, Drouillard JP, Sabatier JC, Tavernier JF. Primary retroperitoneal teratomas in adults. Radiology 1980;134:613-616

6 Ueno T, Tanaka YO, Nagata M, et al. Spectrum of germ cell tumors: from head to toe. Radiographics 2004;24(2):387-404

7 Cho SH, Hong SC, Lee JH, et al. Total laparoscopic resection of primary large retroperitoneal teratoma resembling an ovarian tumor in an adult. J Minim Invasive Gynecol 2008;15(3):384-386

8 Horiuchi A, Watanabe Y, Miyauchi K, et al. Prenatally detected cystic adrenal neuroblastoma. Pediatr Int 2008;50(1):130-131

9 Villarejo F, Scavone C, Blazquez MG, et al. Anterior sacral meningocele: review of the literature. Surg Neurol 1983;19(1):57-71
10 Bakaris S, Senoglu M, Yuksel Z, Gokce M. Lumbosacral neurenteric cyst with associated tethered cord in an adult. Clin Neuropathol 2005;24(2):51-55

11 Cai C, Shen C, Yang W, Zhang Q, Hu X. Intraspinal neurenteric cysts in children. Can J Neurol Sci 2008;35(5):609-615

12 Kumar R, Jain R, Rao KM, Hussain N. Intraspinal neurenteric cystsreport of three paediatric cases. Childs Nerv Syst 2001;17 (10):584-588

13 Choi JY, Kim KN, Kim SE, Yoon DH. Primary malignant fibrous histiocytoma extending into spinal canal through intervertebral foramina. Acta Neurochir (Wien) 2004;146(4): 415-417

14 Jain D, Sharma MC, Sarkar C, et al. Spinal teratoma with renal differentiation-a rare phenomenon: report of two patients. Clin Neurol Neurosurg 2008;110(3):265-269

15 Park SC, Kim KJ, Wang KC, Choe G, Kim HJ. Spinal epidural teratoma: review of spinal teratoma with consideration on the pathogenesis: case report. Neurosurgery 2010;67(6):E1818E1825

16 Jain D, Sharma MC, Sarkar C, et al. Spinal teratoma with pulmonary differentiation: a report of rare case and review of literature. Fetal Pediatr Pathol 2007;26(4):185-191

17 Scott AL, Abbassi-Ghadi N, Archer CM, Swamy R, Gupta S. Neuroendocrine carcinoma arising within a retroperitoneal mature teratoma. Ann R Coll Surg Engl 2010;92(6):W5-8

18 Koen JL, McLendon RE, George TM. Intradural spinal teratoma: evidence for a dysembryogenic origin. Report of four cases. J Neurosurg 1998;89(5):844-851

19 Su TF, Yu CP, Wu ST, et al. Retroperitoneal mixed germ cell tumor mimicking a renal neoplasm: a case report. Urology 2012;80 (3):714-716

20 Uzüm N, Dursun A, Baykaner K, Kurt G. Split-cord malformation and tethered cord associated with immature teratoma. Childs Nerv Syst 2005;21(1):77-80 